\title{
Relative Power of Specific EEG Bands and Their Ratios during Neurofeedback Training in Children with Autism Spectrum Disorder
}

\author{
Yao Wang ${ }^{1,2 *}$, Estate M. Sokhadze ${ }^{3,4,5 *}$, Ayman S. El-Baz ${ }^{3}$, Xiaoli Li $^{1,2 *}$, Lonnie Sears ${ }^{6}$, \\ Manuel F. Casanova ${ }^{4,5}$ and Allan Tasman ${ }^{5}$ \\ 'State Key Laboratory of Cognitive Neuroscience and Learning \& IDG/McGovern Institute for Brain Research, Beijing Normal \\ University, Beijing, China, ${ }^{2}$ Center for Collaboration and Innovation in Brain and Learning Sciences, Beijing Normal University, \\ Beijing, China, ${ }^{3}$ Department of Bioengineering, J.B Speed School of Engineering, University of Louisville, Louisville, KY, USA, \\ ${ }^{4}$ Greenville Health System, Departments of Pediatrics and Biomedical Sciences, University of South Carolina School of \\ Medicine Greenville, Greenville, SC, USA, ${ }^{5}$ Department of Psychiatry and Behavioral Sciences, University of Louisville School \\ of Medicine, Louisville, KY, USA, ${ }^{6}$ Department of Pediatrics, University of Louisville School of Medicine, Louisville, KY, USA
}

\section{OPEN ACCESS}

Edited by: Robert Coben Integrated Neuroscience Services,

Reviewed by: Juliana Yordanova, Institute of Neurobiology, Bulgarian Academy of Sciences, Bulgaria Manousos A. Klados, Max Planck Institute for Human Cognitive and Brain Sciences,

Germany

*Correspondence:

Yao Wang

33048593@qq.com;

Estate M. Sokhadze sokhadze@greenvillemed.sc.edu; Xiaoli Li

xiaoli@bnu.edu.cn

Received: 30 September 2015 Accepted: 24 December 2015 Published: 14 January 2016

Citation:

Wang Y, Sokhadze EM, El-Baz AS,

Li X, Sears L, Casanova MF and Tasman A (2016) Relative Power of Specific EEG Bands and Their Ratios during Neurofeedback Training in Children with Autism Spectrum

Disorder.

Front. Hum. Neurosci. 9:723. doi: 10.3389/fnhum.2015.00723
Neurofeedback is a mode of treatment that is potentially useful for improving self-regulation skills in persons with autism spectrum disorder. We proposed that operant conditioning of EEG in neurofeedback mode can be accompanied by changes in the relative power of EEG bands. However, the details on the change of the relative power of EEG bands during neurofeedback training course in autism are not yet well explored. In this study, we analyzed the EEG recordings of children diagnosed with autism and enrolled in a prefrontal neurofeedback treatment course. The protocol used in this training was aimed at increasing the ability to focus attention, and the procedure represented the wide band EEG amplitude suppression training along with upregulation of the relative power of gamma activity. Quantitative EEG analysis was completed for each session of neurofeedback using wavelet transform to determine the relative power of gamma and theta/beta ratio, and further to detect the statistical changes within and between sessions. We found a linear decrease of theta/beta ratio and a liner increase of relative power of gamma activity over 18 weekly sessions of neurofeedback in 18 high functioning children with autism. The study indicates that neurofeedback is an effective method for altering EEG characteristics associated with the autism spectrum disorder. Also, it provides information about specific changes of EEG activities and details the correlation between changes of EEG and neurofeedback indexes during the course of neurofeedback. This pilot study contributes to the development of more effective approaches to EEG data analysis during prefrontal neurofeedback training in autism.

Keywords: electroencephalography, neurofeedback, autism spectrum disorder, gamma activity, EEG bands' ratios

Abbreviations: ABC, Aberrant Behavior Checklist; ADI-R, Autism Diagnostic Inventory, Revised; ASD, autism spectrum disorder; ADHD, attention deficit hyperactivity disorder; EEG, Electroencephalogram; EMG, Electromyogram; FAI, Focused Attention index; GPI, Gamma power index ( $40 \mathrm{~Hz}$ centered gamma power); NFB, neurofeedback; PAT, Peak Achievement Trainer; rTMS, repetitive Transcranial Magnetic Stimulation; tDCS, transcranial direct current stimulation; WCEC, Weisskopf Child Evaluation Center; WASI, Wechsler Abbreviated Scale of Intelligence; WISC, Wechsler Intelligence Scale for Children. 


\section{INTRODUCTION}

Informed clinical consensus defines autism as a behavioral syndrome characterized by pervasive impairments in several areas of development including social interaction, communication skills, and stereotypical interests and activities (American Psychiatric Association, 2013). Thus, far, there have been no neuropathological findings nor laboratory/performance based measures providing construct validity to the diagnosis. In the absence of pathognomonic abnormalities, clinical research in autism has been guided by a variety of ideologies and epistemological assumptions each contributing to the development of explanatory models or theories: executive function, weak central coherence, complex information processing, theory-of-mind, empathy, anatomical, and functional connectivity, etc. (Baron-Cohen, 1989; Frith and Happé, 1994; Ozonoff et al., 1994; Minshew et al., 1997; Ozonoff, 1997; Belmonte and Yurgelun-Todd, 2003; Belmonte et al., 2004; Baron-Cohen and Belmonte, 2005; Happé and Frith, 2006).

Recent studies by our group have characterized the neuropathology of autism as that of a minicolumnopathy (Casanova et al., 2002a,b, 2003, 2006a,b). Deficits within the inhibitory elements that surround the cell minicolumn suggest a mechanistic explanation to the cortical inhibitory/excitatory (I/E) imbalance in autism (Casanova et al., 2002a,b, 2013; Rubenstein and Merzenich, 2003). Oscillations and synchronization of pyramidal cells in and across minicolumns are maintained by networks of inhibitory GABAergic interneurons (Mann and Paulsen, 2007; Donner and Siegel, 2011). Local I/E interactions shape neuronal representations of sensory, motor, and cognitive variables, and produce local electroencephalographic (EEG) gamma frequency $(30-80 \mathrm{~Hz}$ ) oscillations. The I/E bias caused by faulty pyramidal cellinterneuronal dyads provides a receptive scenario to gamma frequency abnormalities in autism, and can be considered as a neurophysiological, EEG-based biomarker of autism. To the authors' knowledge every study on gamma frequencies in autism has shown abnormalities (Brock et al., 2002; Brown et al., 2005; Pavlova et al., 2006; Orekhova et al., 2007; Rippon et al., 2007; Baruth et al., 2010; Gross et al., 2012; Casanova et al., 2013; Sokhadze et al., 2014).

Strong evidence both from animals and human experiments indicated that high frequency gamma band oscillations, especially those around $40 \mathrm{~Hz}$ frequencies, are most directly associated with entrainment of local networks. Some experimental studies have found that $40 \mathrm{~Hz}$ centered gamma activity may correlate with human perceptual binding (Herrman and Knight, 2001) and attention (Bird et al., 1978). This is especially true for the $35-45 \mathrm{~Hz}$ range gamma sub-band in EEG which was thought to be closely associated with the mechanisms of information processing such as sensory, working memory, attention, and other cognitive processes (Tallon-Baudry et al., 1996; TallonBaudry and Bertrand, 1999; Herrman and Mecklinger, 2000; Grice et al., 2001; Gruber et al., 2001; Tallon-Baudry, 2003). Based on the above, abnormalities in the $35-45 \mathrm{~Hz}$ gamma oscillatory activity probably can be considered as one of the underlying causes of the cognitive deficits observed in autism.
Neurofeedback (NFB) has been recognized as a suitable tool for detecting and modulating neural plasticity due to its ability to non-invasively alter the excitability of neural circuits and for inducing a short-term functional reorganization in anatomically and functionally associated cortical and subcortical neural networks in the human cerebral cortex. By operant conditioning of EEG, NFB provides an effective way to train electrophysiological activity of the targeted cortical area. Multiple NFB studies indicate its usefulness as an efficacious neurotherapy for various mental disorders. Neurofeedback training is considered as one of the most effective and salient treatments for children with attention deficit/hyperactivity disorder (ADHD). The clinical efficacy of using NFB for ADHD treatments was supported by several meta-analyses of randomized clinical trials recently conducted (Lubar, 2003, 2004; Arns et al., 2009; Gevensleben et al., 2009; Sokhadze et al., 2009; Lofthouse et al., 2010). Since many autistic children also show signs of attention-deficit and hyperactivity some attempts have been made to use this technique as a treatment modality for ASD (Linden et al., 1996; Coben and Padolsky, 2007; Coben, 2008, 2013; Kouijzer et al., 2009a,b, 2010; Coben and Myers, 2010; Coben et al., 2010; Sherlin et al., 2010; Thompson et al., 2010a,b; Linden and Gunkelman, 2013). Several current papers review the use of neurofeedback for ASD treatment and many of them provide evidence that some of the core symptoms of autism can be improved this way (Jarusiewicz, 2002; Coben and Padolsky, 2007; Coben, 2008, 2013; Kouijzer et al., 2009a,b; Coben and Myers, 2010; Coben et al., 2010; Sokhadze et al., 2014). During NFB procedure, subjects are trained to enhance desired electro-cortical activity, while suppressing undesirable activity. Through the NFB training course many symptoms related to EEG abnormalities can be corrected and improved toward normalization.

Neurofeedback is relatively new form of treatment for ASD (Kouijzer et al., 2010). There were several case, pilot and group studies (Sichel et al., 1995; Jarusiewicz, 2002) followed by controlled group studies (Coben and Padolsky, 2007; Kouijzer et al., 2009a,b; Coben and Myers, 2010). More detailed accounts summarizing behavioral, cognitive, and neurophysiological data can be found in current reviews (Thompson et al., 2010a,b; Coben, 2013; Linden and Gunkelman, 2013). Among controlled studies should be specifically mentioned quantitative EEG (qEEG) and connectivity analysis guided studies conducted by Coben and his associate (Coben and Padolsky, 2007; Coben and Myers, 2010; Coben et al., 2010; Coben, 2013). QEEG based assessment of functional connectivity is proposed to guide neurofeedback intervention in autism. Some researchers use qEEG-based subtypes or so called endophenotypes to guide neurofeedback in ASD (Linden and Gunkelman, 2013). These techniques use individualized approaches to selection of neurofeedback-based treatment in autism.

QEEG assessments (so called "brain mapping") and training using multiple EEG sensors (usually > 19) is necessary for qEEG-guided neurofeedback. The analysis of current trends in neurofeedback application in ASD shows that multichannel recordings appear to be the way the field is moving for more advanced training models, though for some applications fewer 
channels are required and seem to be sufficient for positive outcomes. In hyperactive individuals, or in a sensory oversensitive autistic child using an EEG cap and preparing skin with abrasive and electrolyte gels may be less feasible. Also some individuals who could really benefit from multichannel EEG recordings and qEEG-guided neurofeedback may not be able to tolerate the EEG caps application and gel in procedures that are used now. Relatively simple to apply one or twochannel wireless neurofeedback systems have certain advantages for neurofeedback training in children with autism.

In the present study we planned to develop methodology to monitor EEG activity and analyze changes during neurofeedback sessions in high-functioning children with ASD. The study represents one of approaches aimed at the understanding of EEG correlates of neurofeedback training in high functioning ASD population, rather than an attempt at claiming clinical improvements resulting from the prefrontal brainwave training. More research studies should be done to understand: (1) whether children with high functioning autism can control EEG in NFB mode, (2) how EEG characteristics are changing during the training course in an ASD population, and (3) what additional efforts are needed to correctly identify specific changes in EEG rhythms known to be abnormal in ASD, specifically gamma activity at the frontal sites.

Our approach included neurofeedback training at the prefrontal topography, specifically at the midline prefrontal site. Considering the role of the prefrontal cortex in executive functions, including attention and cognitive processes, it was feasible to investigate effects of neurofeedback using training at the anterior, frontal location rather than at the central, or posterior (e.g., parietal) sites. This selection of cortical topography was also determined by our prior studies on gamma oscillations in children with autism (Sokhadze et al., 2009; Baruth et al., 2010; Sokhadze, 2012; Casanova et al., 2013) that showed alterations of evoked and induced gamma oscillations during attention tests especially well present at the frontal topographies.

The goal of this study was to conduct neurofeedback in children with ASD using the PAT neurofeedback device with the "Focus/Neureka!" ("Focused Attention" index and " $40 \mathrm{~Hz}$ centered Gamma" index) training protocol of Peak Achievement Trainer (PAT) neurofeedback device (Neurotek, Goshen, KY) to investigate: relative changes in EEG bands (e.g., theta [4$8 \mathrm{~Hz}]$ ) and sub-bands of interest (e.g., low beta [13-18 Hz], high beta $[18-30 \mathrm{~Hz}]$ ) and their ratios (e.g., theta/low beta, etc.) throughout the entire 18 session long course of neurofeedback training in ASD, and during each individual training session using custom-made Matlab application, how gamma power and EEG bands power ratios are changing during individual sessions and between sessions within the course of neurofeedback training in high functioning individuals with ASD, and whether there are any correlation between EEG measures of interest (i.e., relative gamma power, theta/beta ratio) and neurofeedback training indices such as "Focused Attention" index and " $40 \mathrm{~Hz}$-centered Gamma" index (Cowan and Albers, 2008).

It was expected that all participants would complete 18 weekly sessions of $\sim 25-30 \mathrm{~min}$ long training and learn to increase the "Focused Attention" measure, and control level of
" $40 \mathrm{~Hz}$ centered Gamma" parameter in neurofeedback mode. It was similarly expected that an increase in so called "Focused Attention" measure of the PAT device protocol would be manifested in a gradual decrease of theta/low beta and theta/high beta EEG ratios, while an increase in the " $40 \mathrm{~Hz}$ centered Gamma" measure would be accompanied by the gradual increase of the relative power of gamma $(30-45 \mathrm{~Hz})$ band.

\section{METHODS}

\section{Patient Demographics and Recruitment}

Eighteen children and adolescents with ASD (mean age 13.2 years, $\mathrm{SD}=4.3,4$ females, 14 males) were recruited in this study. It was not required for participants to be off medication during the whole course of the neurofeedback trainings. Medication status, dosage, and other variables of pharmacotherapy were accurately monitored and recorded, but were not used as a part of the patients' demographic descriptive characteristics in this study. Before the neurofeedback session, on the days of their visit, all participants were requested not to take medication. Participants with ASD were recruited through the University of Louisville Weisskopf Child Evaluation Center (WCEC). All participants with ASD were diagnosed by an experienced pediatrician according to the Diagnostic and Statistical Manual of Mental Disorders (DSM-IV-TR) (American Psychiatric Association, 2000) and further ascertained with the Autism Diagnostic Interview-Revised (ADI-R, Le Couteur et al., 2003). Further, medical estimations were made to exclude the participants with a history of seizure, significant hearing, or visual impairment, a brain abnormality or an identified genetic disorder. Participants with severe psychiatric comorbidities were not included in the study. All patients were naive to neurofeedback training procedures and never participated in any neurofeedback study before.

Using the Wechsler Intelligence Scale for Children (WISC-IV, Wechsler, 2004) or (for adolescents) the Wechsler Abbreviated Scale of Intelligence (WASI) (Wechsler, 1999), all participants were assessed to have full-scale IQ $>80$. Fourteen participants were high-functioning persons with autism diagnosis and four had Asperger Syndrome. Child and adolescent psychiatrist and clinical psychologist at the WCEC performed pre- and postneurofeedback clinical evaluations. Neurofeedback sessions were conducted by an experienced applied psychophysiologist. All required IRB-approved consent/assent forms were signed by the participants and their parents/guardians.

\section{Behavioral Measures and Evaluation}

In conjunction with the EEG data we collected the behavioral rating results with the pre- and post-neurofeedback data using the Aberrant Behavior Checklist (ABC) (Aman and Singh, 1994) from the parents of the ASD participants. Irritability, Lethargy/Social Withdrawal, Stereotypy, Hyperactivity, and Inappropriate Speech were the five problem aspects that were contained in and assessed by the $\mathrm{ABC}$ rating scale. In the current study, we focus on the Hyperactivity, Lethargy, and Irritability ratings before and after a course of NFB treatments. 


\section{Neurofeedback Protocol and Data Collection}

In the study, ASD participants completed a course of NFB trainings using a "Focus/Neureka!" protocol of the PAT neurofeedback device designed to modulate the "Focused Attention" index (FAI) and " $40 \mathrm{~Hz}$-centered Gamma" index (GPI). The prefrontal neurofeedback training protocol used in this study was based on the BioExplorer software (CyberEvolution, Seattle, WA, USA) platform. The protocol provided the exercises for each subject to enhance the singlepointed "Focused Attention" index measure (FAI) throughout the session while maintaining an adequate level of "Neureka!" measure (GPI) within a certain range. During all of the treatment sessions different scenes from the BBC "Planet Earth" and "Life" series were shown to maintain the participants' adherence. The protocol in this study provides feedback to the subjects in both visual and auditory modalities. Based on the thresholds set, parameters related with visual feedback such as the brightness, size, and continuation of the video have been modulated and the sound volume of the video adjusted simultaneously according to the "FAI" and "GPI" measures during the treatment. All EEG signals and training parameters were measured using three electrodes, one active electrode at the prefrontal EEG (FPz) site, the second being a reference on the left ear, and a third sensor serving as ground and located between the two above electrodes. All of the subjects in the study were requested to complete a $25-30 \mathrm{~min}$ recording per session and a total of 18 weekly neurofeedback sessions, in order to increase the "FAI" and "GPI" using the "Focus/Neureka!" PAT protocol. More than $90 \%$ of the sessions met the requirement of a 20 -min minimum usable EEG data recording. Eye blink and EMG artifacts removal was implemented using the specific BioExplorer application that can be found in the operation manual of the NFB device.

\section{The EEG Signal Processing}

The EEG signal collected and recorded by BioExplorer applications during NFB treatments were exported and further analyzed by a series of customized codes using Matlab software (MathWorks, Inc, Massachusetts). As an extension application of BioExplorer software, BioReview report can be called to export the raw EEG and the desired frequency bands of data for each session. By configurations in the BioReview report, along with the raw EEG, the separated delta $(2-4 \mathrm{~Hz})$, theta $(4-8 \mathrm{~Hz})$, alpha $(8-$ $13 \mathrm{~Hz})$, low beta $(13-18 \mathrm{~Hz})$, high beta $(18-30 \mathrm{~Hz})$, and gamma $(30-45 \mathrm{~Hz})$ were also acquired using 7 th order elliptical bandpass filters. The exported data have been arranged in a text file in which the different items were organized into columns and each subsequent row represented the data point in time series between samples.

For the relative power calculation, it was necessary to gain the total power of the band from 2 to $45 \mathrm{~Hz}$ (the whole bands from delta to gamma frequencies). A custom band-pass filter application integrated of wavelet transformation and a Harris window configuration were created to filter and separate the 2$45 \mathrm{~Hz}$ frequency band from the raw signal that was exported from the BioReview reports. The wavelet analysis was used to provide enhanced temporal resolution of frequency responses of a given signal and it allowed us to apply a band pass filter to the individual waveform and avoid the distortion when applying the filter to the entire signal. In the study, the sample-rate of the raw signals collected in the Bioexplorer system was $256 \mathrm{~Hz}$. The EEG changes in the prefrontal site during each session for all subjects ( $20 \mathrm{~min}$ data per session and 18 sessions for each subject) were analyzed in Matlab. The continuous wavelet transformation of the signal is shown in the Equation below, in which

$S($ scale $)=1 /$ frequency; $\tau=$ time $\operatorname{shift} ; \operatorname{Psi}(\psi)=$ mother wavelet(in our case the Morlet window).

$$
C W T_{\chi}^{\psi}(\tau, s)=\Psi_{\chi}^{\psi}(\tau, s)=\frac{1}{\sqrt{s}} \int \chi(t) \psi^{*}\left(\frac{t-\tau}{s}\right) d t
$$

In our codes, the Morlet window was used to separate the raw signal (the first column in the text file exported from the Bioexplorer) into 128 coefficients. And the coefficients then were filtered into $2-45 \mathrm{~Hz}$ frequency by applying the Harris window.

The equation for the 4-term Harris window of length $N$ is shown below.

$$
\begin{array}{r}
\omega(\mathrm{n})=a_{0}-a_{1} \cos \left(\frac{2 \pi n}{N-1}\right)+a_{2} \cos \left(\frac{4 \pi n}{N-1}\right)-a_{3} \cos \left(\frac{6 \pi n}{N-1}\right) \\
a_{0}=0.355768 ; a_{1}=0.487396 ; a_{2}=0.144232 ; a_{3}=0.012604 ;
\end{array}
$$

The Harris window is employed for convolution with each coefficient. It provides a good method to avoid the leakage of the high variable dynamic EEG signals. At last, the filtered coefficients were transformed and summed to produce a reconstructed filtered signal (Figure 1).

Besides the relative power calculation for each band, the ratio of certain bands was also calculated. The formula used to calculate relative power is given in the equation below, where $B$ represents the band's signal and $\mathcal{T}$ represents the total signal $(2-45 \mathrm{~Hz})$.

$$
\sum_{0}^{i} \mathcal{B}^{2} / \sum_{0}^{i} \mathcal{T}^{2}
$$

The ratios of interest for this study were theta $(4-8 \mathrm{~Hz})$ to low beta $(13-18 \mathrm{~Hz})-$ Theta/low beta ratio, theta to high beta $(18-$ $30 \mathrm{~Hz}$ ) - Theta/high beta ratio, and cumulative theta to beta (13-30 Hz) ratio (Theta/beta).

\section{Statistical Analysis}

The primary statistical analyses in the study mainly included linear regression estimation and paired sample $t$-test methods. Each EEG dependent variable over 18 sessions of neurofeedback course was analyzed using linear regression analyses and the mean values of dependent EEG variables at the first and last session of the NFB course together with the pre- and post-NFB behavioral measures using $\mathrm{ABC}$ questionnaire were compared with the paired sample $t$-test method. EEG variables and "FAI" and "GPI" NFB training indices were calculated as well on per minute basis during each 


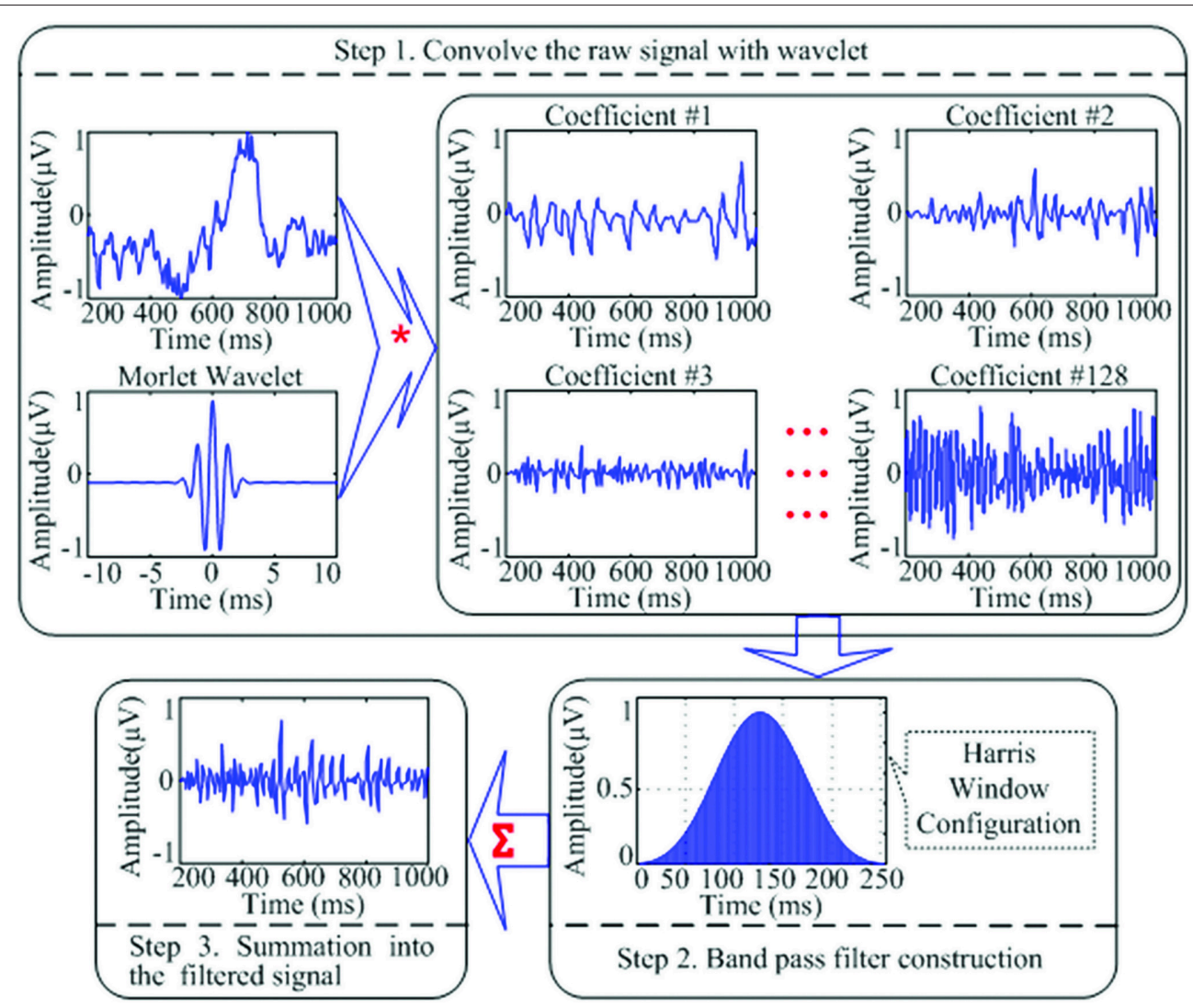

FIGURE 1 | A schematic representation of the wavelet transformation and band-pass filtering applications utilized to filter raw EEG signal into the desired filtered signal to be used for relative power calculation of the EEG bands of interest. "*” Means the process of "convolution"; " $\sum$ " means the process of "summation"; the three lines of "..." are the ellipsis points, which means in the similar fashion from "Coefficient \#3" to "Coefficient \#128."

training session. Each dependent EEG variable went through the normality distribution analysis using $t$-test to ensure appropriateness for the test, and $95 \%$ confidence intervals (95\% CI) were included in outcomes. Pearson correlation analysis was used for individual EEG measures, neurofeedback training indices, and behavioral measures collected using ABC questionnaire.

\section{RESULTS}

\section{EEG Activity Measures across 18 Sessions of Neurofeedback}

Relative power of gamma activity (power within $35-45 \mathrm{~Hz}$ vs. total power in $2-45 \mathrm{~Hz}$, in percentage) showed statistically significant linear increase over 18 sessions of neurofeedback (linear regression: $R=0.491, R^{2}=0.241, y=0.022 \mathrm{x}+$ $1.45 \%, t=2.25, p=0.039$, power of test 0.55 at $\alpha=0.05$, below the desired level of 0.80, Figure 2, Table 1). However, paired sample Student's $t$-test showed that relative power of gamma did not increase statistically from the first to the last neurofeedback session [from $1.57 \pm 1.30 \%$ to $1.80 \pm 1.15 \%$, mean increase $0.23 \pm 0.29 \%, t_{(17)}=0.76, p=0.456$, n.s., Table 2].

Theta/low beta ratio showed a statistically significant linear decrease over 18 sessions of neurofeedback $\left(R=0.666, R^{2}=\right.$

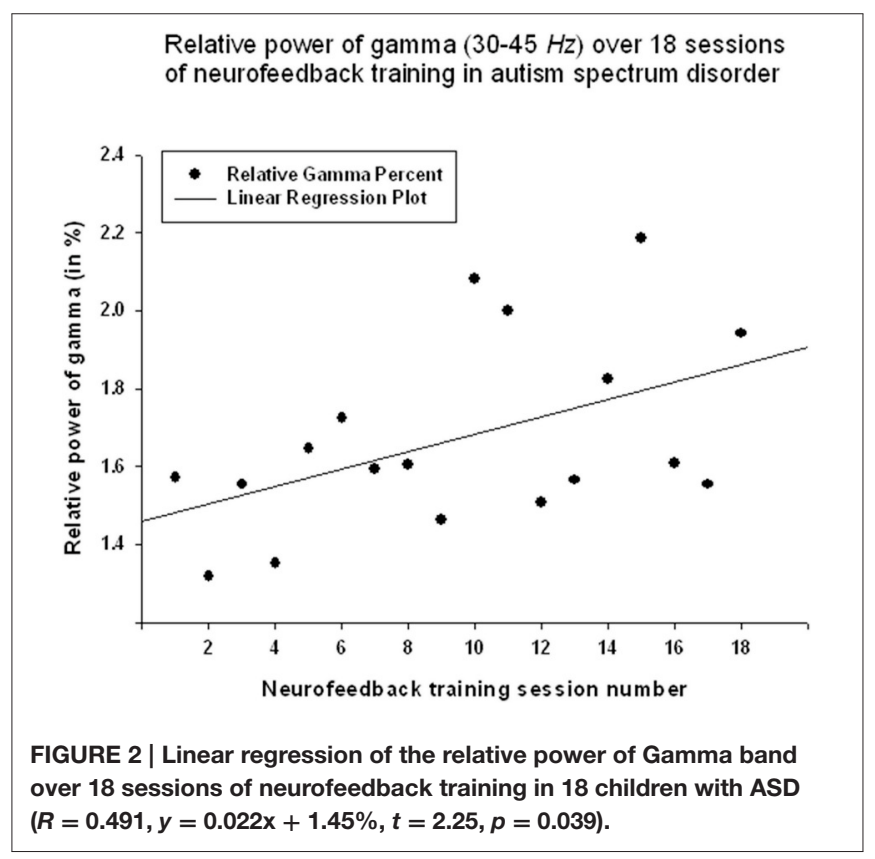

$0.444, y=-0.079 \mathrm{x}+9.49, t=-3.57, p=0.003$, power $=0.87$, Figure 3 ) and $t$-test showed that theta/low beta ratio decreased statistically from the first to the last neurofeedback session [from 
TABLE 1 | Summary of linear regression statistics for main dependent variables in 18 sessions of NFB.

\begin{tabular}{|c|c|c|c|c|c|c|c|}
\hline Measures & Units & $t$ & $P$-value & $\boldsymbol{R}$ & $R^{2}$ & Regression equation & Power \\
\hline Gamma & $\%$ & 2.25 & 0.039 & 0.491 & 0.241 & $y=0.022 x+1.45$ & 0.548 \\
\hline Theta/low beta & N/A & -3.57 & 0.003 & 0.666 & 0.444 & $y=-0.079 x+9.49$ & 0.876 \\
\hline Theta/high beta & $\mathrm{N} / \mathrm{A}$ & -4.01 & 0.001 & 0.708 & 0.502 & $y=-0.088 x+6.26$ & 0.928 \\
\hline "Focus Attention" index & C.U. & 1.84 & 0.084 & 0.418 & 0.175 & $y=0.056 x+73.83$ & 0.408 \\
\hline "40 Hz Gamma" index & C.U. & 2.61 & 0.019 & 0.547 & 0.299 & $y=0.165 x+42.37$ & 0.662 \\
\hline
\end{tabular}

TABLE 2 | Paired sample $t$-test of the last vs. first neurofeedback session in 18 subjects.

\begin{tabular}{|c|c|c|c|c|c|c|c|c|}
\hline \multirow[t]{3}{*}{ EEG measures Last-minus-first } & \multirow[t]{3}{*}{ Units } & \multicolumn{4}{|c|}{ Paired differences } & \multirow[t]{3}{*}{$\boldsymbol{t}$} & \multirow[t]{3}{*}{ df } & \multirow[t]{3}{*}{$P$-value } \\
\hline & & \multirow[t]{2}{*}{ Mean } & \multirow[t]{2}{*}{ Std. Dev. } & \multicolumn{2}{|c|}{$95 \% \mathrm{Cl}$} & & & \\
\hline & & & & Lower & Upper & & & \\
\hline Gamma & $\%$ & 0.22 & 1.24 & -0.84 & 0.39 & 0.76 & 17 & 0.456 \\
\hline Theta/low beta & N/A & -1.72 & 3.40 & 0.032 & 3.42 & -2.15 & 17 & 0.046 \\
\hline Theta/high beta & N/A & -1.48 & 2.83 & 0.081 & 2.89 & -2.23 & 17 & 0.039 \\
\hline Theta/beta & N/A & -1.26 & 2.47 & 0.033 & 2.49 & -2.16 & 17 & 0.045 \\
\hline "Focused Attention" index & C.U. & 2.29 & 2.25 & -3.41 & -1.17 & 4.32 & 17 & 0.001 \\
\hline "40 Hz-centered Gamma" index & C.U. & 3.68 & 6.66 & -7.00 & -0.37 & 2.34 & 17 & 0.031 \\
\hline
\end{tabular}

$9.54 \pm 3.57$ to $7.81 \pm 1.46$, mean decrease being $-1.72 \pm 3.40$, $\left.t_{(17)}=-2.15, p=0.046\right]$. Regression of the theta/high beta ratio over 18 sessions showed a significant linear correlation $\left[R=0.708, R^{2}=0.502, y=-0.088 \mathrm{x}+6.24, t_{(17)}=-4.01\right.$, $p=0.001$, power $=0.92$, Figure 4]. $T$-test showed a significant decrease from the first to the last session [from $6.22 \pm 3.11$ to $\left.4.73 \pm 2.16, t_{(17)}=-2.23, p=0.039\right]$. The ratio of theta/beta (i.e., theta ratio to sum of low and high beta, 13-30 Hz) showed a similar decrease trend $\left[-1.26 \pm 2.47, t_{(17)}=-2.16, p=\right.$ 0.045].

\section{Neurofeedback Training Indices}

The "Focused Attention" index (FAI, i.e., "Inhibit All" measure in neurofeedback) did not show a statistically significant linear increase over 18 sessions of training $\left[R=0.418, R^{2}=0.175\right.$, $t_{(18)}=1.84, p=0.084$, n.s.], but $t$-test showed significant changes from the first to the last session of neurofeedback [from $73.1 \pm 4.85$ to $75.39 \pm 5.27$ c.u., $\left.t_{(17)}=4.32, p=0.001\right]$. The other neurofeedback measure reflecting relative power of " $40-\mathrm{Hz}$ centered Gamma" index did show a linear increase trend over 18 sessions of training $\left[R=0.547, R^{2}=0.299, t_{(17)}=2.61, p=\right.$ 0.019 , power $=0.66$, Figure 5$]$ and paired sample $t$-test confirmed that the change of this index from the first to the last session was statistically significant [from $42.34 \pm 7.45$ to $46.03 \pm 6.13$ c.u., $\left.t_{(17)}=2.34, p=0.031\right]$. This neurofeedback index showed a significant positive Pearson correlation coefficient with relative gamma power across 18 session of training $(r=0.548, p=$ 0.019). On the other hand, the "Focused Attention" index showed a negative correlation with the theta/low beta ratio $(r=-0.51$, $p=0.03)$ and with the theta/beta ratio $(r=-0.59, p=0.01)$ across 18 sessions of neurofeedback.

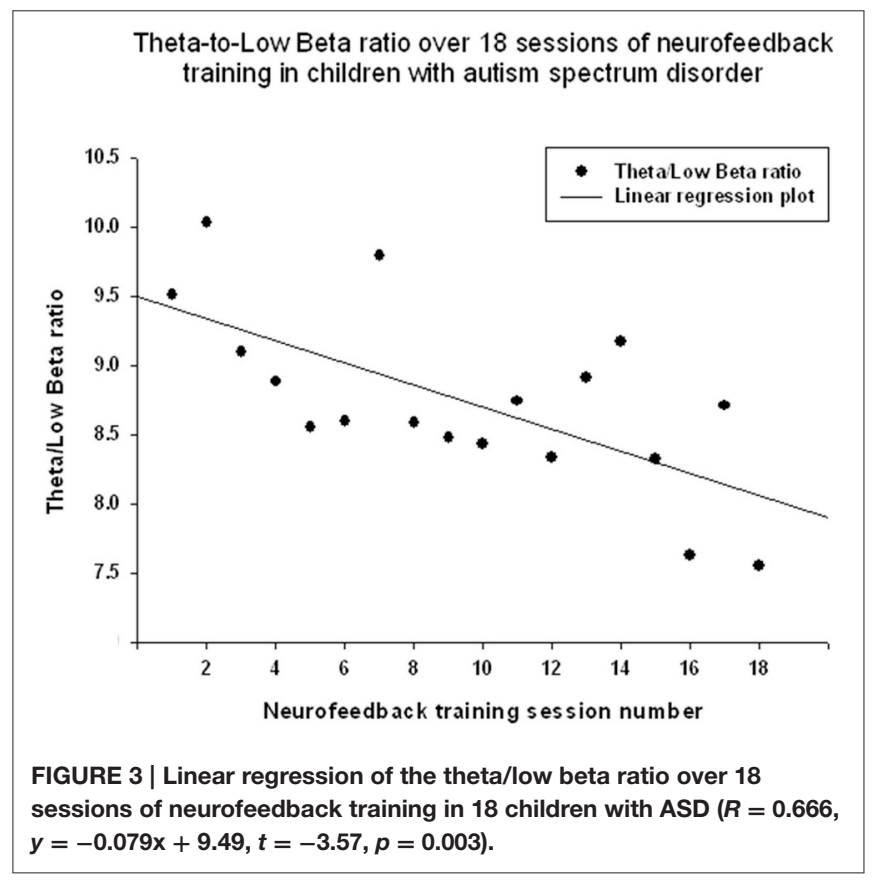

\section{EEG and Neurofeedback Training Measures during 20 Min Session}

EEG measures did not show a significant linear regression over 20 min of neurofeedback session. The "Focused Attention" index did show a statistically significant linear increase during each neurofeedback session $\left[R=0.576, R^{2}=0.332, y=0.063 \mathrm{x}+\right.$ 70.64 c.u., $t_{(19)}=2.99, p=0.008$, power $=0.77$ at $\alpha=0.05$, 

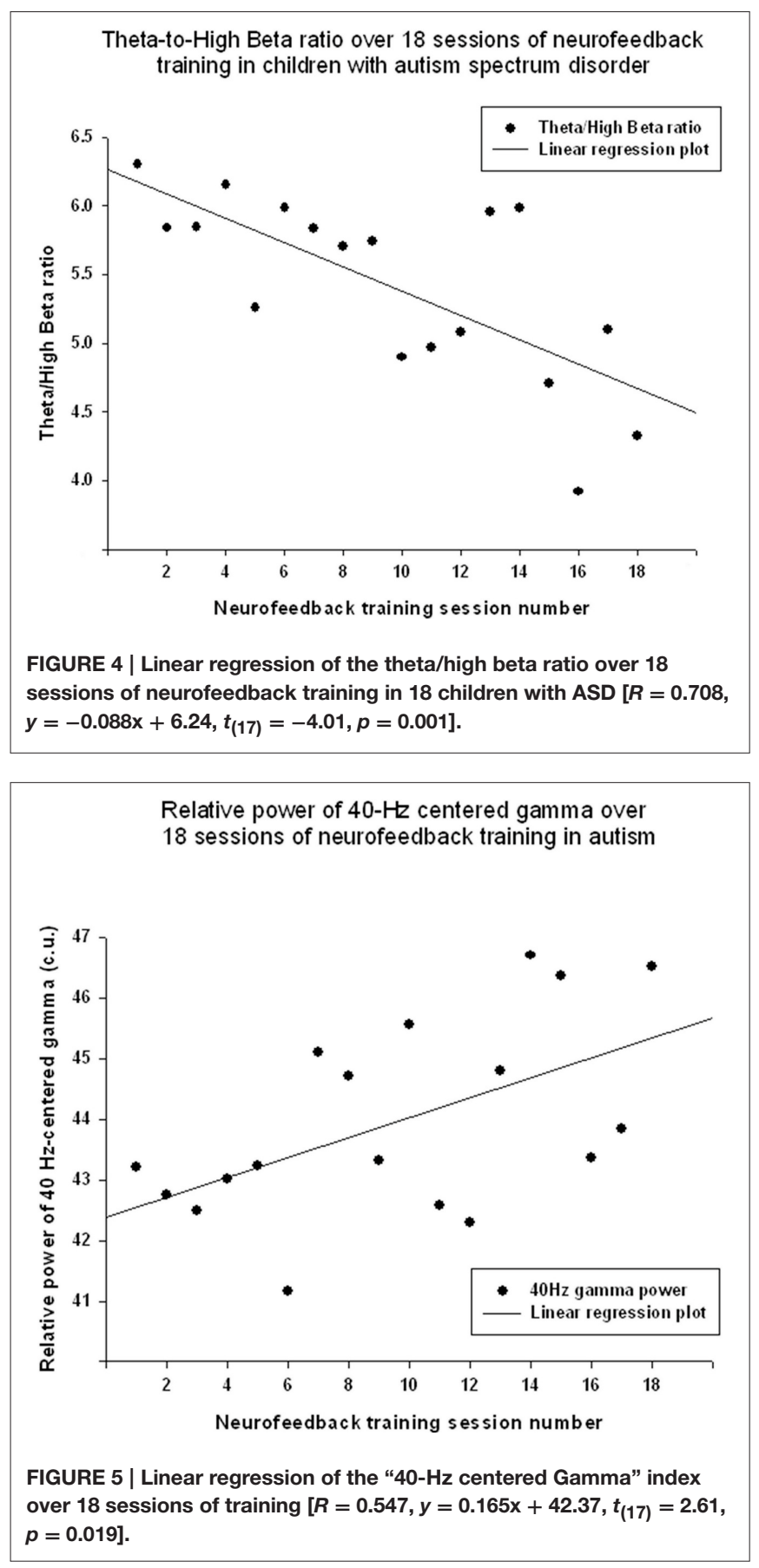

Figure 6]. This index had a significant negative correlation with the theta/beta ratio during $20 \mathrm{~min}$ long neurofeedback sessions ( $r=-0.70, p=0.001$, Figure 7 ). It should be noted that the theta/low beta and theta/high beta ratios showed a high level positive correlation during training sessions $(r=0.63, p=$ $0.003)$.

\section{Behavioral Evaluations}

There was a significant reduction in Lethargy/Social Withdrawal subscale of the ABC. The rating scores showed a reduction [from

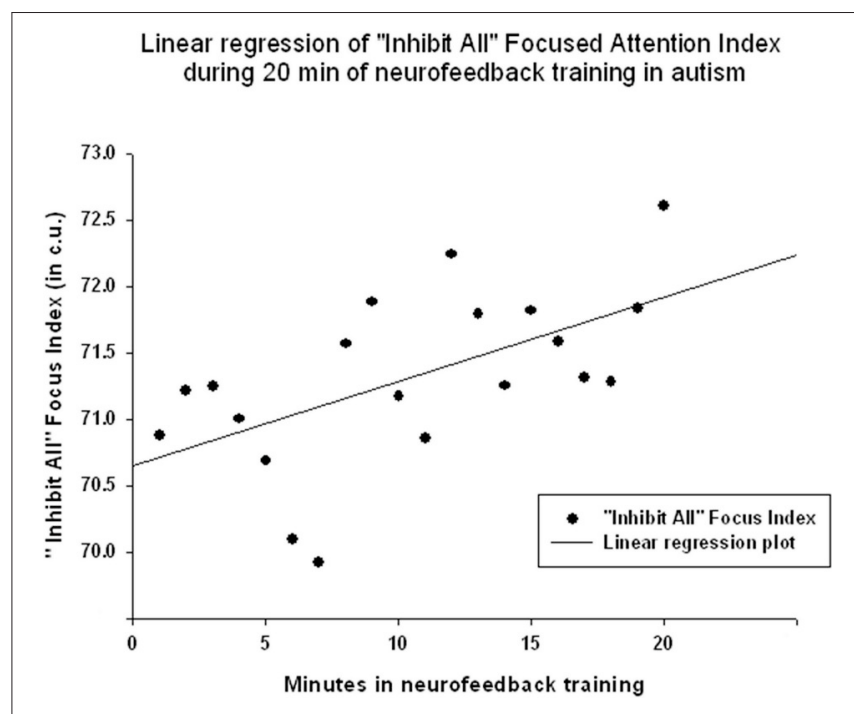

FIGURE 6 | Linear regression of the "Focused Attention" index during $\mathbf{2 0}$ min long neurofeedback session (mean for all subjects across all sessions $\left[R=0.576, y=0.063 x+70.64\right.$ c.u., $\left.t_{(19)}=2.99, p=0.008\right]$.

Correlation of Theta-to-Beta power ratio with "Inhibit All" Focused Attention Index during 20 min of neurofeedback training (all sessions)

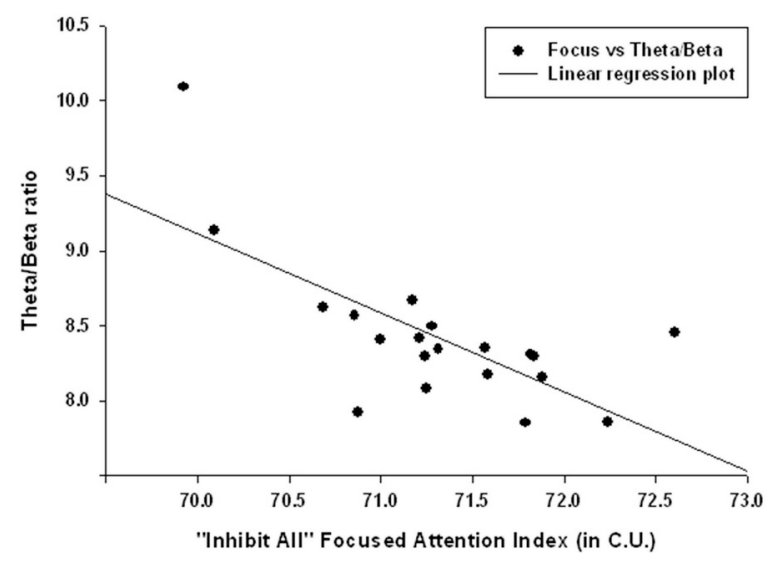

FIGURE 7 | Correlation of the theta/beta with the "Focused Attention" index during $\mathbf{2 0} \mathrm{min}$ of neurofeedback training (across all sessions, $r=-0.70, p=0.001$ ).

$10.18 \pm 6.07$ to $7.53 \pm 5.82$, change was $-2.64 \pm 3.13, t_{(17)}=$ $3.29, p=0.005$ ], while Hyperactivity scores also showed decrease [from $16.65 \pm 13.78$ to $13.29 \pm 11.97,-3.35 \pm 5.39, t_{(17)}=2.56$, $p=0.021]$.

Changes in Lethargy/Social Withdrawal scores showed a positive correlation with relative gamma power changes $(r=0.43, p=0.041)$, and negative correlation with the theta/low beta $(r=-0.43, p=0.043)$ and theta/high beta ratios $(r=-0.45, p=0.033)$. Hyperactivity scores on $\mathrm{ABC}$ did not show any statistically significant correlation with EEG 
measures (i.e., theta/beta ratio, gamma) or NFB indices (i.e., "Focus" index, " $40 \mathrm{~Hz}$ Gamma" index). Changes on other rating scales of the $\mathrm{ABC}$ did not reach a significance level. For instance, Irritability rating showed a trend to decrease from $9.59 \pm 7.65$ to $8.17 \pm 7.13$, changing by $-1.41 \pm 4.33, t_{(17)}=1.35, p=0.198$, n.s. Change of Stereotypy was only $-0.88 \pm 2.97, t_{(17)}=1.22$, $p=0.239$. Changes in Inappropriate Speech scores were even smaller $(-0.29 \pm 1.86, p=0.524)$.

\section{DISCUSSION}

The results indicate that the study outcomes were very close to the predicted ones, especially in regards to changes in the ratios of interest (theta/low beta, theta/high beta, theta/Beta) from the first to the last session, and regression of the dependent variables across the neurofeedback training sessions. For instance, the theta/beta showed a decrement across NFB sessions (Figure 3) while the relative power of the gamma band showed a linear increase over the course of the training (Figure 2). Both neurofeedback training indices ("Focused Attention" index and " $40 \mathrm{~Hz}$ Gamma" index) showed a linear increase over training sessions and increased significantly toward the end of the course. We could not find, however, significant trends of the EEG variables changes within the 20 min of individual neurofeedback sessions. Only one training index (i.e., "Focused Attention," Figure 6) showed a linear increase over the minutes within individual sessions and high negative correlation with both theta/low beta and theta/high beta ratios in the EEG (Figure 7).

We found a notable decrease in the theta/low beta and theta/high beta proportions from session to session along with an increase of both training indices to the end of the course. These results are in accordance with the goals of NFB treatment described earlier for children with ADHD (Arns et al., 2009; Lofthouse et al., 2010). Even though the theta/low beta ratios used in prior ADHD NFB studies were mostly collected from the central cortical sites (e.g., $\mathrm{Cz}$ ), our frontal theta/low beta ratios showed similar trends in ADHD population (Hillard et al., 2013; Sokhadze et al., 2013). In this study, a reduction in the theta/beta proportions at the prefrontal site was robust across sessions. Due to the improvements in behavioral outcomes indicated by $\mathrm{ABC}$ questionnaire, it is also possible to discuss whether training of the "Focus/40 Hz Gamma" measures of the PAT protocol are related to functional behavioral improvements reported by the patients. Determining which of these two measures is more fundamental to the effects of neurofeedback in ASD would increase the efficiency and aid in the delivery of more effective neurofeedback treatment methods.

As mentioned earlier, autism is characterized by an imbalanced inhibitory/excitatory ratio in local cortical network, which may cause the disordered gamma oscillations in ASD reflected at the electroencephalographic level. The gamma abnormalities and excessive cortical excitation (E/I ratio) in autism have been considered as an important EEG biomarkers for ASD based on recent theoretical reviews and experimental studies (Casanova et al., 2015; Uzunova et al., 2015). Brown et al. (2005) interpreted the abnormal gamma responses in their study on individuals with autism as reflecting decreased "signal to noise" ratio due to decreased inhibitory processing (Grice et al., 2001; Lansbergen et al., 2011). Brock et al. (2002) described the parallels between the psychological model of "central coherence" (Frith and Happé, 1994) in information processing and their neuroscience model of neural integration or "temporal binding" (Szentagothai and Arbib, 1975). This concept was further elaborated in an "impaired connectivity" hypothesis of autism which summarized theoretical and empirical advances in research implicating disordered connectivity in autism (Brown, 2005). The authors highlighted recent developments in the analysis of the temporal binding of information and the relevance of gamma activity to current models of structural and effective connectivity based on the balance between excitatory and inhibitory cortical activity (Casanova et al., 2002b, 2013; Belmonte and Yurgelun-Todd, 2003; Belmonte et al., 2004; Rippon et al., 2007). Based on the minicolumn hypothesis of autism, disrupted patterns of coordinated high frequency oscillatory output in distributed networks might be associated with cortical "disconnection" in autism according to Casanova et al. (2006a,b).

The current study indicated the effectiveness of prefrontal neurofeedback aimed at modulating the disordered EEG activities associated with ASD. Also, from the results of the correlation between " $40 \mathrm{~Hz}$ Gamma" index and the relative power of gamma calculated in our custom made program, the "Focus/40 Hz Gamma" protocol provided by the neurofeedback equipment used in our study can effectively help to improve gamma activity along with the decrement of theta/beta ratio in prefrontal EEG in children with ASD (Sokhadze et al., 2009; Casanova et al., 2015). Further, the results of the relative power of gamma and the band ratios that were calculated in our wavelet transform based program show us more details of how ASD subjects were controlling their EEG during the NFB treatments. Exported from the NFB software, the raw EEG data were filtered and calculated in our own program developed in the study. The Wavelet transformation based program could provide us the enhanced temporal resolution of frequency responses of a given signal and help to acquire the accurate and intact dynamic information. As a non-linear time-varying signal, EEG frequency data are suitable for being analyzed by Wavelet transformation algorithms. The custom made codes developed in the study provide us an important off-line method for clearly detecting the specific changing characteristics of EEG activities during the NFB treatments.

Theta/beta ratios (both theta/low beta and theta/high beta) showed the significant linear decrease over 18 sessions of neurofeedback, and in addition $t$-test showed that the ratios decreased statistically from the first to the last NFB training sessions. Theta/beta ratio is one of the classical indices for characterizing the ability to focus attention and to concentrate. The current study showed that both prefrontal theta/beta ratio and power of gamma activity could be modulated positively by operant conditioning during the NFB training in high functioning children with ASD. It is well-known that most ASD subjects have difficulties with switching focused attention. The "Focus $/ 40 \mathrm{~Hz}$ Gamma" protocol used in the study provided a successful way for positively modulating both gamma activity 
and focused concentration abilities in ASD. The positive effects of the neurofeedback training further can be manifested by the improvement in the behavioral scores measured by the $\mathrm{ABC}$. Our results show a significant reduction in the Lethargy/Social Withdrawal subscale of the $\mathrm{ABC}$ and a negative correlation with the theta/beta ratio. The Hyperactivity scores of $\mathrm{ABC}$ also showed a decrease but the same did not correlate with any EEG or NFB indices used in this study. The improvement of behavioral changes assessed by $\mathrm{ABC}$ before and after the 18 sessions of NFB treatments was in accordance to the functional outcomes seen in the EEG profile changes. Our study showed that compared to previous protocols that required more sessions per subject ( $>30$ ) and a more frequent training rate (e.g., twice per week), the statistical significant improvement either in EEG or in behavioral measures (Sokhadze et al., 2009) can also be achieved within a shorter number of sessions (i.e., $18 \mathrm{NFB}$ sessions in ASD, or even 12 sessions in ADHD, Hillard et al., 2013) and weekly visits. Probably more than 18 sessions might contribute to better consolidation of results of operant conditioning using neurofeedback, and currently we have studies in progress that will compare outcomes of 12 vs. 18 vs. 24 sessions of neurofeedback using the same protocol in children with autism. Our future efforts will be directed to combine the neurofeedback with other novel neuromodulation techniques employed in autism treatment (e.g., rTMS, tDCS, auditory integration training, etc.).

EEG oscillations in the gamma band, and specifically those centered around $40 \mathrm{~Hz}$ has been historically associated with feature binding and cognitive processes (Tallon-Baudry et al., 1996), and neurofeedback studies targeted at changing $40 \mathrm{~Hz}$ gamma power popular back in 70s and 80s (Bird et al., 1978) are still attracting interest of investigators (Keizer et al., 2010). One of the important characteristics of our version of $40 \mathrm{~Hz}$ centered gamma power upregulation was integration of gamma training with the task of maintaining focused attention index. Another important feature of the protocol specifics was recording gamma activity at the midline prefrontal site. One more specific feature of the protocol used in this study was weekly training sessions that allowed subjects to stay longer in treatment. However, the major difference from other studies using neurofeedback in autism was in careful analysis of the dynamics of EEG bands of interest (i.e., theta, beta, and gamma) and their ratios during individual

\section{REFERENCES}

Aman, M. G., and Singh, N. N. (1994). Aberrant Behavior Checklist-Community. Supplementary Manual. East Aurora, NY: Slosson Educational Publications.

American Psychiatric Association (2000). Diagnostic and Statistical Manual of Mental Disorders, 4th Edn. Washington, DC: American Psychiatric Publishing.

American Psychiatric Association (2013). Diagnostic and Statistical Manual of Mental Disorders, 5th Edn. Arlington, VA: American Psychiatric Publishing.

Arns, M., de Ridder, S., Strehal, U., Breteler, M., and Coenen, A. (2009). Efficacy of neurofeedback treatment in ADHD: the effects on inattention, impulsivity and hyperactivity: a meta-analysis. Clin. EEG Neurosci. 40, 180-189. doi: 10.1177/155005940904000311

Baron-Cohen, S. (1989). The theory of mind hypothesis of autism: a reply to Boucher. Br. J. Disord. Commun. 24, 199-200. doi: $10.3109 / 13682828909011956$ neurofeedback sessions and across the whole 18 sessions-long course of treatment. The study provided additional solution to analysis of EEG during neurofeedback training using custommade Matlab application.

It should be noted that the study has several limitations. The enrollment to the neurofeedback training was open to only highfunctioning children with autism and children with Asperger syndrome, thus results cannot be directly interpolated for low functioning children with ASD. The study was not designed as a clinical research as it had no control group of participants, and the number of clinical behavioral evaluations was minimal. The focus of current study was directed toward more accurate analysis of EEG signal using custom-made software with the aim of exploration of the dynamics of EEG activity during the neurofeedback training course in children with autism. Records of patients demographic specifics (e.g., social status of families, ASD onset, and duration data, etc.) and detail of their medication status were not analyzed.

In order to foster the neurofeedback treatment applications for children with ASD and its scientific rationale, further methodological advances are necessary: controlled and randomized study designs, larger sample sizes of patients, a more accurate selection of subjects with ASD, and more intensive and rigorous baseline, post-treatment- and follow-up evaluations.

\section{AUTHOR CONTRIBUTIONS}

For the manuscript, YW is responsible for the data processing and writing; Prof. ES mainly focused on the experiments design and the whole procedures; Prof. AE developed the program in Matlab; Prof. XL help to review the Matlab codes; Dr. LS mainly responsible for the behavioral assessments for ASD subjects during the experiments; Prof. MC partially provided the financial supports for the whole study; Prof. AT reviewed the manuscript and did many modifications for it.

\section{ACKNOWLEDGMENTS}

The study was supported by China Scholarship Council (CSC) grant to Ph.D. candidate YW (No.201306040059). 
Bird, B. F., Newton, A. D., Sheer, E., and Ford, M. (1978). Behavioral and electroencephalographic correlates of $40 \mathrm{~Hz}$ EEG biofeedback training in humans. Biofeedback Self Regul. 3, 13-28. doi: 10.1007/BF00998560

Brock, J., Brown, C. C., Boucher, J., and Rippon, G. (2002). The temporal binding deficit hypothesis of autism. Dev. Psychopathol. 14, 209-224. doi: 10.1017/S0954579402002018

Brown, C., Gruber, T., Boucher, J., Rippon, G., and Brock, J. (2005). Gamma abnormalities during perception of illusory figures in autism. Cortex 41, 364-376. doi: 10.1016/S0010-9452(08)70273-9

Brown, C. (2005). "EEG in autism: is there just too much going on in there?", in Recent Developments in Autism Research, ed M. F. Casanova (New York, NY: Nova Science Publishers), 109-126.

Casanova, M. F., Buxhoeveden, D. P., and Brown, C. (2002a). Clinical and macroscopic correlates of minicolumnar pathology in autism. J. Child Neurol. 17, 692-695. doi: 10.1177/088307380201700908

Casanova, M. F., Buxhoeveden, D. P., Switala, A. E., and Roy, E. (2002b). Minicolumnar pathology in autism. Neurology 58, 428-432. doi: 10.1212/WNL.58.3.428

Casanova, M. F., Buxhoeveden, D., and Gomez, J. (2003). Disruption in the inhibitory architecture of the cell minicolumn: implications for autism. Neuroscientist 9, 496-507. doi: 10.1177/1073858403253552

Casanova, M. F., van Kooten, I. A., Switala, A. E., van Engeland, H., Heinsen, H., Steinbusch, H. W., et al. (2006a). Minicolumnar abnormalities in autism. Acta Neuropathol. 112, 287-303. doi: 10.1007/s00401-006-0085-5

Casanova, M. F., van Kooten, I. A., Switala, E., van England, H., Heinsen, H., Steinbuch, H. W. M., et al. (2006b). Abnormalities of cortical minicolumnar organization in the prefrontal lobes of autistic patients. Clin. Neurosci. Res. 6, 127-133. doi: 10.1016/j.cnr.2006.06.003

Casanova, M. F., Baruth, J. A., El-Baz, S., Sokhadze, G. E., Hensley, M., and Sokhadze, E. M. (2013). "Evoked and induced gamma- frequency oscillation in autism," in Imaging the Brain in Autism, eds M. Casanova, A. El-Baz, and J. S. Suri (New York, NY: Springer), 87-106.

Casanova, M. F., Sokhadze, E., Opris, I., Wang, Y., and Li, X. (2015). Autism spectrum disorders: linking neuropathological findings to treatment with transcranial magnetic stimulation. Acta Pediatr. 104, 346-355. doi: 10.1111/apa.12943

Coben, R., and Padolsky, J. (2007). Assessment-guided neurofeedback for autistic spectrum disorders. J. Neurother. 11, 5-23. doi: 10.1300/J184v11n01_02

Coben, R. (2008). Autistic spectrum disorder: a controlled study of EEG coherence training focused on social skills deficits. J. Neurother. 12, 57-75.

Coben, R., and Myers,T. E. (2010). The relative efficacy of connectivity guided and symptom based EEG biofeedback for autistic disorders. Appl. Psychophysiol. Biofeedback. 35, 13-23. doi: 10.1007/s10484-009-9102-5

Coben, R., Linden, M., and Myers, T. E. (2010). Neurofeedback for autistic spectrum disorder: a review of the literature. Appl. Psychophysiol. Biofeedback. 35, 83-105. doi: 10.1007/s10484-009-9117-y

Coben, R. (2013). "Neurofeedback for autistic disorders: emerging empirical evidence," in Imaging the Brain in Autism, eds M. F. Casanova, A. S. El-Baz, and J. S. Suri (New York, NY: Springer), 107-134.

Cowan, J. D., and Albers, S. (2008). Manual for the Peak Achievement Trainer. Goshen, KY: NeuroTek LLC. Available online at: http://www.peakachievement. com/homeuser/Peak_Achievement_Trainer.htm

Donner, T. H., and Siegel, M. (2011). A framework for local cortical oscillation patterns. Trends Cogn. Sci. 15, 191-199. doi: 10.1016/j.tics.2011.03.007

Frith, U., and Happé, F. (1994). Autism: beyond theory of mind. Cognition 50, 115-132. doi: 10.1016/0010-0277(94)90024-8

Gevensleben, H., Holl, B., Albrecht, B., Vogel, C., Schlamp, D., Kratz, O., et al. (2009). Is neurofeedback an efficacious treatment for ADHD? A randomised controlled clinical trial. J. Child Psychol. Psychiatry 50, 780-789. doi: 10.1111/j.1469-7610.2008.02033.x

Grice, S. J., Spratling, M. W., Karmiloff-Smith, A., Halit, H., Csibra, G., de Haan, M., et al. (2001). Disordered visual processing and oscillatory brain activity in autism and Williams syndrome. NeuroReport 12, 2697-2700. doi: 10.1097/00001756-200108280-00021

Gross, E., El-Baz, A. S., Sokhadze, G. E., Sears, L., Casanova, M. F., and Sokhadze, E. M. (2012). Induced EEG gamma oscillation alignment improves differentiation between autism and ADHD group responses in a facial categorization task. J. Neurother. 16, 78-91. doi: 10.1080/10874208.2012.677631
Gruber, T., Keil, A., and Müller, M. M. (2001). Modulation of induced gamma band responses and phase synchrony in a paired associate learning task in the human EEG. Neurosci. Lett. 316, 29-32. doi: 10.1016/S0304-3940(01)02361-8

Happé, F., and Frith, U. (2006). The weak coherence account: detail-focused cognitive style in autism spectrum disorders. J. Autism Dev. Disord. 36, 5-25. doi: 10.1007/s10803-005-0039-0

Herrman, C. S., and Mecklinger, A. (2000). Magnetoencephalographic responses to illusory figures: early evoked gamma is affected by processing of stimulus features. Int. J. Psychophysiol. 38, 265-281. doi: 10.1016/S0167-8760(00)0 0170-7

Herrman, C. S., and Knight, R. T. (2001). Mechanisms of human attention: event related potentials and oscillations. Neurosci. Biobehav. Rev. 25, 465-476. doi: 10.1016/S0149-7634(01)00027-6

Hillard, B., El-Baz, A. S., Sears, L., Tasman, A., and Sokhadze, E. M. (2013). Neurofeedback training aimed to improve focused attention and alertness in children with ADHD: a study of relative power of EEG rhythms using custom-made software application. Clin. EEG Neurosci. 44, 193-202. doi: $10.1177 / 1550059412458262$

Jarusiewicz, B. (2002). Efficacy of neurofeedback for children in the autistic spectrum: a pilot study. J. Neurother. 6, 39-49. doi: 10.1300/J184v06n04_05

Keizer, A. W., Verschoor, M., Verment, R. S., and Hommel, B. (2010). The effect of gamma enhancing neurofeedback on the control of feature binding and intelligence measures. Int. J. Psychophys. 75, 25-32. doi: 10.1016/j.ijpsycho.2009.10.011

Kouijzer, M., de Moor, J. M. H., Gerrits, B. J., Congedo, M., and van Schie, H. (2009a). Neurofeedback improves executive functioning in children with autism spectrum disorders. Res. Aut. Spectr. Disord. 3, 145-162. doi: 10.1016/j.rasd.2008.05.001

Kouijzer, M., de Moor, J., Gerrits, B., Buitelaar, J., and van Schie, H. (2009b). Longterm effects of neurofeedback treatment in autism. Res. Aut. Spectr. Disord. 3, 496-501. doi: 10.1016/j.rasd.2008.10.003

Kouijzer, M., van Schie, H., de Moor, J. M. H., Gerrits, B. J., and Buitelaar, J. K. (2010). Neurofeedback treatment in autism: preliminary findings in behavioral, cognitive, and neurophysiological functioning. Res. Aut. Spectr. Disord. 4, 386-399. doi: 10.1016/j.rasd.2009.10.007

Lansbergen, M. M., Arns, M., van Dongen-Boomsma, M., Spronk, D., and Buitelaar, J. K. (2011). The increase in theta/beta ratio on resting-state EEG in boys with attention-deficit/hyperactivity disorder in mediated by slow alpha peak frequency. Prog. Neuropsychopharm. Biol. Psychiatry 35, 47-52. doi: 10.1016/j.pnpbp.2010.08.004

Le Couteur, A., Lord, C., and Rutter, M. (2003). The Autism Diagnostic InterviewRevised (ADI-R). Los Angeles, CA: Western Psychological Services.

Linden, M., Habib, T., and Radojevic, V. (1996). A controlled study of the effects of EEG biofeedback on cognition and behavior of children with attention deficit disorder and learning disabilities. Biofeedback Self Regul. 21, 35-49. doi: 10.1007/BF02214148

Linden, M., and Gunkelman, J. (2013). "QEEG-guided neurofeedback for autism: clinical observations and outcomes," in Imaging the Brain in Autism, eds M. F. Casanova, A. S. El-Baz, and J. S. Suri (New York, NY: Springer), 45-60.

Lofthouse, N., Arnold, L. E., and Hurt, E. (2010). A comment on Sherlin, Arns, Lubar, and Sokhadze. J. Neurother. 14, 301-306. doi: $10.1080 / 10874208.2010 .523340$

Lubar, J. F. (2003). "Neurofeedback for the management of attention deficit disorders," in The Biofeedback: A Practitioner's Guide, $3 r d$ Edn, eds M. Schwartz and F. Andrasik (New York, NY: Guilford Press), 409-437.

Lubar, J. F. (Ed.). (2004). Quantitative Electroencephalographic Analysis (QEEG) Databases for Neurotherapy: Description, Validation, and Application. New York, NY: Haworth Press, Inc.

Mann, E. O., and Paulsen, O. (2007). Role of GABAergic inhibition in hippocampal network oscillations. Trends Neurosci. 30, 343-349. doi: 10.1016/j.tins.2007.05.003

Minshew, N. J., Goldstein, G., and Siegel, D. J. (1997). Neuropsychologic functioning in autism: profile of a complex information processing disorder. J. Int. Neuropsychol. Soc. 3, 303-316.

Orekhova, E. V., Stroganova, T. A., Nygren, G., Tsetlin, M. M., Posikera, I. N., Gillberg, C., et al. (2007). Excess of high frequency electroencephalogram oscillations in boys with autism. Biol. Psychiatry 62, 1022-1029. doi: 10.1016/j.biopsych.2006.12.029 
Ozonoff, S. (1997). "Casual mechanisms of autism: unifying perspectives from an information-processing framework," in Handbook of Autism and Pervasive Developmental Disorders, eds F. R. Volkmar, R. Paul, A. Klin, and D. J. Cohen (New York, NY: John Wiley), 868-879.

Ozonoff, S., Stayer, D. L., McMahon, W. M., and Filloux, F. (1994). Executive function abilities in autism and Tourette syndrome: an information processing approach. J. Child Psychol. Psychiatry 35, 1015-1132. doi: 10.1111/j.14697610.1994.tb01807.x

Pavlova, M., Birbaumer, N., and Sokolov, A. (2006). Attentional modulation of cortical neuromagnetic gamma response to biological movement. Cereb. Cortex 16, 321-327. doi: 10.1093/cercor/bhi108

Rippon, G., Brock, J., Brown, C., and Boucher, J. (2007). Disordered connectivity in the autistic brain: challenges for the "new psychophysiology." Int. J. Psychophysiol. 63, 164-172. doi: 10.1016/j.ijpsycho.2006.03.012

Rubenstein, J. L., and Merzenich, M. M. (2003). Model of autism: increased ratio of excitation/inhibition in key neural systems. Genes Brain Behav. 2, 255-267. doi: 10.1034/j.1601-183X.2003.00037.x

Sherlin, L., Arns, M., Lubar, J., and Sokhadze, E. (2010). A position paper on neurofeedback for the treatment of ADHD. J. Neurother. 14, 66-78. doi: 10.1080/10874201003773880

Sichel, A. G., Fehmi, L. G., and Goldstein, D. M. (1995). Positive outcome with neurofeedback treatment in a case of mild autism. J. Neurother. 1, 60-64. doi: 10.1300/J184v01n01 08

Sokhadze, E. M., El-Baz, A., Baruth, J., Mathai, G., Sears, L., and Casanova, M. F. (2009). Effect of a low frequency repetitive transcranial magnetic stimulation(rTMS) on gamma frequency oscillations and event-related potentials during processing of illusory figures in autism. J. Aut. Dev. Disord. 39, 619-634. doi: 10.1007/s10803-008-0662-7

Sokhadze, E. (2012). Peak performance training using prefrontal EEG biofeedback. Biofeedback 40, 7-15. doi: 10.5298/1081-5937-39.3.4

Sokhadze, E. M., Hillard, B., El-Baz, A. S., Tasman, A., and Sears, L. (2013). Electroencephalographic biofeedback improves focused attention in attention deficit/hyperactivity disorder. Bull. Siber. Med. 12, 182-194.

Sokhadze, E. M., El-Baz, A. S., Tasman, A., Sears, L., Wang, Y., Lamina, E. V., et al. (2014). Neuromodulation integrating rTMS and neurofeedback for the treatment of autism spectrum disorder: an exploratory study. Appl. Psychophysiol. Biofeedback 39, 237-225. doi: 10.1007/s10484-014-9264-7
Szentagothai, J., and Arbib, M. A. (1975). Conceptual Models of Neural Organization. Boston, MA: MIT Press.

Tallon-Baudry, C., Bertrand, O., Delpuech, C., and Pernier, J. (1996). Stimulus specificity of phase-locked and non-phase-locked $40 \mathrm{~Hz}$ visual responses in human. J. Neurosci. 16, 4240-4249.

Tallon-Baudry, C., and Bertrand, O. (1999). Oscillatory gamma activity in humans and its role in object representation. Trends Cogn. Sci. 3, 151-162. doi: 10.1016/S1364-6613(99)01299-1

Tallon-Baudry, C. (2003). Oscillatory synchrony and human visual cognition. J. Physiol. Paris 97, 355-363. doi: 10.1016/j.jphysparis.2003.09.009

Thompson, L., Thompson, M., and Reid, A. (2010a). Functional neuroanatomy and the rationale for using EEG biofeedback for clients with Asperger's syndrome. Appl. Psychophysiol. Biofeedback 35, 39-61. doi: 10.1007/s10484009-9095-0

Thompson, L., Thompson, M., and Reid, A. (2010b). Neurofeedback outcomes in clients with Asperger's syndrome. Appl. Psychophysiol. Biofeedback 35, 63-81. doi: 10.1007/s10484-009-9120-3

Uzunova, G., Pallanti, S., and Hollander, E. (2015). Excitatory/inhibitory imbalance in autism spectrum disorders: implications for interventions and therapeutics. World J. Biol. Psychiatry. doi: 10.3109/15622975.2015.1085597. [Epub ahead of print].

Wechsler, D. (1999). Wechsler Abbreviated Scale of Intelligence. San Antonio, TX: Harcourt Assessment, Inc.

Wechsler, D. (2004). Wechsler Intelligence Scale for Children-Fourth Edition Integrated (WISC-IV Integrated). San Antonio, TX: Harcourt.

Conflict of Interest Statement: The authors declare that the research was conducted in the absence of any commercial or financial relationships that could be construed as a potential conflict of interest.

Copyright (c) 2016 Wang, Sokhadze, El-Baz, Li, Sears, Casanova and Tasman. This is an open-access article distributed under the terms of the Creative Commons Attribution License (CC BY). The use, distribution or reproduction in other forums is permitted, provided the original author(s) or licensor are credited and that the original publication in this journal is cited, in accordance with accepted academic practice. No use, distribution or reproduction is permitted which does not comply with these terms. 